\title{
HYPOCOMPLEMENTEMIC URTICARIFORM VASCULITIS CASE REPORT: COMPLETE REMISSION AFTER INFUSION OF RITUXIMAB IN A REFERENCE HOSPITAL OF SÃO PAULO
}

Sandra Patricia Echazú Román ${ }^{1, *}$, Luiza Trincado ${ }^{1}$, Renata Maria Monteiro Pinto ${ }^{1}$, Marina de Azevedo Martins ${ }^{1}$, Danielle Arraes Rubini ${ }^{1}$, Renata Ferreira Rosa ${ }^{1}$, Rina Dalva Neubarth Giorgi ${ }^{1}$

1. Instituto de Assistência ao Servidor Público Estadual', São Paulo (SP), Brazil.

*Corresponding author: sandra_echazu@hotmail.com

\section{INTRODUCTION}

Urticarial vasculitis is a small vessel vasculitis presented with urticarial lesions instead of palpable purpura. Hypocomplementemic vasculitis is a severe form of disease with extracutaneous manifestations including obstructive pulmonary disease (50\%), uveitis, episcleritis, fever, angioedema, peripheral neuropathy, pericarditis, transient migratory large joints arthritis, recurrent abdominal pain, glomerulonephritis and seizures. Laboratories abnormalities include antic1q antibodies, low c3c4 complement, elevated ESR, positive ANA and RF. Patients with positive ANA, SLE e Sjogren's syndrome should be investigated as a primary cause. Involvement tends to be on the trunk and distal lower extremities. Often resolved hyperpigmentation, lesions characterized by pain, burning and associated symptoms fever, arthralgia, abdominal pain. Histology: leukocytoclastic vasculitis. Treatment with NSAIDs, prednisone, dapsone or hydroxychloroquine. Patients with severe disease may use cyclophosphamide, azathioprine, mycophenolate mofetil. Rituximab is an alternative treatment followed by iv immunoglobulin in refractory disease.

\section{CASE REPORT}

A 56-year-old patient, female, with clinical condition of urticarial vasculitis initializing in 2013 in lower distal extremities, referred pain and burning. Previous symptoms were fever, fatigue, weight loss and arthralgia. Requested serology for $B$ and $C$ hepatitis, HIV results negative, paraneoplastic investigation negative with endoscopy and colonoscopy due to increase CA19.9. In 2013, she performed a biopsy of the lesion with results of leukocytoclastic vasculitis, positive laboratories results were C-ANCA, low c3c4 complement, $\mathrm{RF}+$, ANA 1/160 reticulate pointed cytoplasmatic, C1q negative in 2018. Patient treatment was refractory to the use of prednisone with the necessity of pulse therapy in two occasions due to the vasculitis lesions in 2017 and twice in 2018. Treatment: Azathioprine was used in 2018 until 2019, with secondary effects, dapsone and hydroxychloroquine posterior to that, without control of the disease. Presenting the necessity of the introduction of rituximab $500 \mathrm{mg}$ weakly during 4 weeks in 2019. Due to pandemic of coronavirus, a new infusion of rituximab was delayed and observed remission of the lesions after 1 year and 3 months.

\section{CONCLUSION}

Hypocomplementemic urticariform vasculitis is a syndrome that can be usually easily resolved, but there are severe cases with necessity of immunosuppressor, high doses prednisone, even immunosuppressor due to refractory treatment. Despite the lack of literature due to few reported cases and after therapeutic failure with most variety of immunosuppressors, we chose to treat our patient with rituximab, reasoned by the physiopathological base of the disease, achieving remission and good outcome with success. This case report support evidence for future studies with Rituximab treatment of hypocomplementemic urticariform vasculitis. 